\title{
Steady-State Response of Periodically Switched Linear Circuits via Augmented Time-Invariant Nodal Analysis
}

\author{
Riccardo Trinchero, Igor S. Stievano, and Flavio G. Canavero \\ Department of Electronics and Telecommunications, Politecnico di Torino, Corso Duca degli Abruzzi, 24, 10129 Torino, Italy \\ Correspondence should be addressed to Riccardo Trinchero; riccardo.trinchero@polito.it
}

Received 9 May 2014; Revised 15 July 2014; Accepted 31 July 2014; Published 2 September 2014

Academic Editor: Muhammad Taher Abuelma'atti

Copyright ( 2014 Riccardo Trinchero et al. This is an open access article distributed under the Creative Commons Attribution License, which permits unrestricted use, distribution, and reproduction in any medium, provided the original work is properly cited.

\begin{abstract}
We focus on the simulation of periodically switched linear circuits. The basic notation and theoretical framework are presented, with emphasis on the differences between the linear time-invariant and the time-varying cases. For this important class of circuits and sources defined by periodic signals, the computation of their steady-state response is carried out via the solution of an augmented time-invariant MNA equation in the frequency-domain. The proposed method is based on the expansion of the unknown voltages and currents in terms of Fourier series and on the automatic generation of augmented equivalents of the circuit components. The above equivalents along with the information on circuit topology allow creating, via circuit inspection, a time-invariant MNA equation, the solution of which provides the coefficients of both the time- and the frequency-domain responses of the circuit. Analytical and numerical examples are used to stress the generality and benefits of the proposed approach.
\end{abstract}

\section{Introduction}

Nowadays, a number of alternative methods and tools for the analysis of time-varying circuits and systems that exhibit a periodic switching behavior have been developed and are currently available to designers. They are used to predict both the time- and the frequency-domain responses of electrical and electronic circuits that are characterized by the possibly periodic activity of internal switches during the initial design phase. Well-known examples are the power converters that are massively used to energize almost any equipment or appliance.

A seminal contribution to circuit analysis has been proposed by Zadeh and Desoer in $[1,2]$ where the basic definitions and mathematical tools are presented as the natural extension of the results available for the linear time-invariant (LTI) case [3]. In the 70s, Liou developed a closed-form solution for the complete frequency-domain response of periodically switched linear circuits [4]. Based on the above results, Strom and Signell [5] extended the previous works to more complex networks with an arbitrary number of switches. The common background of the above contributions is the analysis of a circuit by means of a state-equation formalism, intended to compute the socalled generalized transfer functions and impulse responses of time-varying circuits. These approaches, however, require a strong mathematical development and heavy numerical computations. Yuan and Opal $[6,7]$ introduced an innovative simulation approach via the classical modified nodal analysis (MNA) tool. In the latter contribution, the circuits are frozen and solved in all the different operating states of the switches. The dynamical effects of switches on the circuit solution are then taken into account via a suitable coupling involving the dynamical elements within the net. More recently, other techniques such as Floquet theory [8], truncated harmonic balance [9], and time-invariant multifrequency (TIMFS) model [10] have been proposed in order to study and to simulate linear periodically time-varying systems both in frequency- and in time-domains. Without loss of generality, the readers should also refer to [11, 12] where additional properties of time-varying systems are available and to [1315] where applications are presented.

The present contribution extends the results of previously published papers in this field and provides an alternative approach to the steady-state analysis of periodically switched linear (PSL) circuits. The proposed method is based on 
the generation of augmented time-invariant characteristics of the PSL elements directly in frequency-domain via the harmonic balance technique and on the computation of steady-state response of a PSL circuit by means of the standard solution of an augmented linear time-invariant nodal equation generated from circuit inspection only. The proposed solution dramatically reduces the mathematical complexity and offers a modular approach to circuit analysis, leading to accurate results with remarkable simulation speedup. The paper collects a brief overview of the essential theory needed to handle PSL circuits in order to provide a selfcontained document allowing the readers to readily apply the proposed methodology to their own application problems. To this aim, simple analytical examples and topological intuition are used to demonstrate the generality of the method. Its feasibility and strength are instead provided by a real application example involving a switching power converter in [16]. The proposed approach is used to predict and to understand the conducted emission of switching devices, improving the state-of-the-art results $[17,18]$. In the above assessment, the conducted disturbances are quantified by means of the frequency spectrum of the steady-state response of the current supplying the converter.

The remaining part of the paper is organized as follows. Section 2 outlines the basic notation and definitions of LTI systems. Their generalization to the case of time-varying circuits is included in Section 3. Section 4 focuses on the important class of periodically switched circuits and illustrates the basic constitutive operators and rules governing their behavior. The core of the proposed method is presented in Sections 5 and 6 where augmented characteristic equations of resistive PSL elements are derived and used to generate a time-invariant MNA equation describing a generic PSL circuit. Specific emphasis is given to a topological approach for the construction of the advocated augmented nodal equations in matrix form. Section 7 collects the results of the application of the proposed solution to two example circuits, with additional details on the accuracy and efficiency of the method. Summary and conclusions are given in Section 8 .

\section{Linear Time-Invariant Systems}

This section summarizes the standard symbols and definitions used in this paper. A generic single-input single-output LTI system [3] is defined by the following compact notation:

$$
y_{\text {out }}(t)=L\left[x_{\text {in }}(t)\right]
$$

where $x_{\text {in }}$ and $y_{\text {out }}$ are the input and the output signals, respectively, and $L$ is the operator or rule accounting for the system behavior.

For a system defined by (1), the evolution of the output signal can be computed from the impulse response $h(t)$ by means of the convolution integral that writes

$$
y_{\text {out }}(t)=h(t) * x_{\text {in }}(t)=\int_{-\infty}^{+\infty} h(t-\tau) x_{\text {in }}(\tau) d \tau
$$

where $h(t)=L[\delta(t)], \delta(t)$ being the Dirac delta function. Additionally, the well-known counterpart of (2) in the frequency-domain writes

$$
Y_{\text {out }}(\omega)=H(\omega) X_{\text {in }}(\omega),
$$

where $H(\omega)$ is the frequency-domain network function obtained via the Fourier transform of the impulse response $h(t)$; that is,

$$
H(\omega)=\int_{-\infty}^{+\infty} h(t) \exp (-j \omega t) d t
$$

\section{Linear Time-Varying Circuits}

This section collects the essential theoretical results needed to extend the characterization of LTI systems to the more complex case of time-varying circuits. The readers should refer to [1] for a more exhaustive and in-depth treatment. Here, the discussion is aimed at presenting the notation and the main mathematical tools allowing handling these systems.

For the sake of illustration, the example of Figure 1 is considered. It shows a simple time-varying circuit consisting of two resistors, one capacitor, and one switch (Figure 1(a)) and its system representation in terms of interconnected blocks (Figure 1(b)). From the above example, clearly, the output of the system $y_{\text {out }}$ depends on the position of the switch $S$. For a fixed state of $S$, the output can be defined by either $h_{1}(t)$ or $h_{2}(t)$ and the standard convolution integral given by (2) can be used to compute the system response. However, to account for a possible time-varying activity of the system, (2) must be replaced by

$$
y_{\text {out }}(t)=\int_{-\infty}^{+\infty} h(t, \tau) x_{\text {in }}(\tau) d \tau
$$

where the generalized impulse response $h(t, \tau)$ is defined by $h(t, \tau)=L[\delta(t-\tau)]$ (where in a causal linear time-varying system $h(t, \tau)=0$ for $t<\tau)$ according to [1,11]. It is relevant to remark that, in the previous equation, $t$ plays the role of the observation time and $\tau$ represents the so-called excitation time accounting for the position of the delta function feeding the system. In other words, the excitation time is used to explore the time-varying nature of the system under different operating conditions.

The dependence of the generalized impulse response on the additional variable $\tau$ requires the definition of the bifrequency transfer function $H(\omega, \Omega)$ that can be computed by the impulse response via the following integral:

$$
H(\omega, \Omega)=\iint_{-\infty}^{+\infty} h(t, \tau) \exp (-j(\omega t-\Omega \tau)) d t d \tau .
$$

The above equation extends the Fourier transform of the classical impulse response $h(t)$ to the two-dimensional case $h(t, \tau)$ (see [1] for additional details). Similarly, the generalization of (3) becomes

$$
Y_{\text {out }}(\omega)=\frac{1}{2 \pi} \int_{-\infty}^{+\infty} H(\omega, \Omega) X_{\text {in }}(\Omega) d \Omega
$$




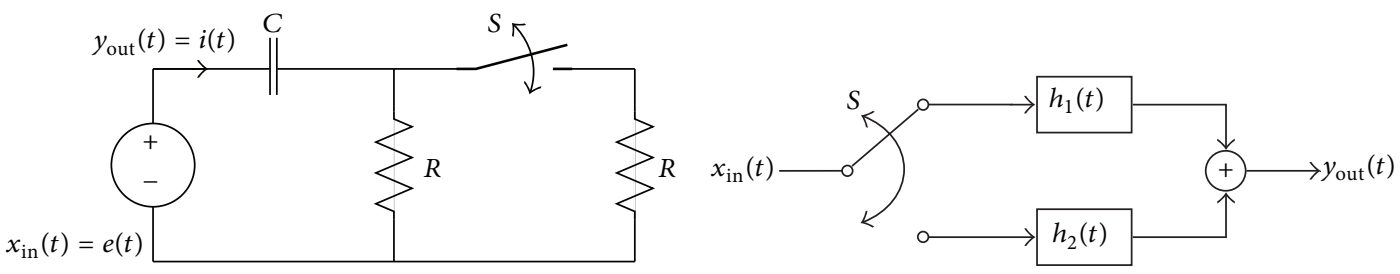

(a)

(b)

FIGURE 1: (a) Example time-varying circuit; (b) system equivalent defined in terms of two LTI blocks (viz. $h_{1}$ and $h_{2}$ ) and one switch.

From the previous result, the bifrequency transfer function $H(\omega, \Omega)$ can be considered as a map between the input $X_{\text {in }}(\Omega)$ and the output $Y_{\text {out }}(\omega)$, where the variables $\Omega$ and $\omega$ are the input and output frequencies, respectively.

Equation (7) suggests that a sinusoidal excitation with angular frequency $\Omega$ feeding the system produces an output response characterized by a possibly richer frequencydomain behavior (i.e., $Y_{\text {out }}$ turns out to be a function of $\omega$ ). On the contrary, it is well known that LTI systems operating in the sinusoidal steady-state at $\Omega$ produce responses characterized by the same input frequency. In the latter case, $Y_{\text {out }}$ would consist of two delta functions located at $\omega= \pm \Omega$.

In this framework, an important role is played by the timevarying transfer function:

$$
H(t, \Omega)=\int_{-\infty}^{+\infty} h(t, \tau) \exp (-j \Omega(t-\tau)) d \tau .
$$

It relates the time-domain response of the system $y_{\text {out }}(t)$ to the corresponding frequency-domain excitation $X_{\text {in }}(\omega)$, where the $\omega$ variable needs to be replaced by $\Omega$, leading to

$$
y_{\text {out }}(t)=\frac{1}{2 \pi} \int_{-\infty}^{+\infty} H(t, \Omega) X_{\text {in }}(\Omega) \exp (j \Omega t) d \Omega .
$$

Based on [11], that provides a summary of direct and inverse transformations involving the above defined operators; the bifrequency transfer can be alternatively obtained from (8) via

$$
H(\omega, \Omega)=\int_{-\infty}^{+\infty} H(t, \Omega) \exp (j(\Omega-\omega) t) d t .
$$

The above equations constitute the basic set of definitions needed to solve both analytically and numerically timevarying circuits. This overview also highlights the main differences between time-invariant and time-varying systems. In the latter case, the relations among the input and output variables in both time- and frequency-domain involve more complex operators rather than simple products and convolutions. The mathematics is unavoidably more complex. However, the framework is well established and allows us to effectively employ the readily available results to real application examples.

\section{Periodically Switched Linear Circuits}

This section deals with the analysis of PSL circuits by means of the generalized transfer functions described in the previous section. A PSL circuit can be seen as a particular case of a time-varying linear system, where the circuit configuration changes periodically in time due to the effect of the switches. The example of Figure 1 becomes a PSL circuit when the position of the switch $S$ is periodically changed between the close and the open states. It is important to remark that this class of circuits represents an important subset of time-varying systems with a large number of applications in different engineering domains.

For PSL circuits, the time-varying transfer function $H(t, \Omega)(8)$ turns out to be a periodic function of $t$ that can be expanded in Fourier series, leading to [6]

$$
H(t, \Omega)=\sum_{n=-\infty}^{+\infty} H_{n}(\Omega) \exp \left(j n \omega_{c} t\right),
$$

where $\omega_{c}=2 \pi / T$ is the fundamental angular frequency of the switch ( $T$ being the characteristic period) and $H_{n}(\Omega)$ are new objects called aliasing transfer functions, defined as

$$
H_{n}(\Omega)=\frac{1}{T} \int_{0}^{T} H(t, \Omega) \exp \left(-j n \omega_{c} t\right) d t .
$$

Substituting (11) into (10) gives

$$
H(\omega, \Omega)=2 \pi \sum_{n=-\infty}^{+\infty} H_{n}(\Omega) \delta\left(\omega-\Omega-n \omega_{c}\right) .
$$

Hence, the output $Y_{\text {out }}(\omega)$ in frequency-domain can finally be computed via (7) and writes

$$
Y_{\mathrm{out}}(\omega)=\sum_{n=-\infty}^{+\infty} H_{n}\left(\omega-n \omega_{c}\right) X_{\mathrm{in}}\left(\omega-n \omega_{c}\right)
$$

The above equation is the generalization of (3) for linear periodically switched systems. This can be appreciated by observing that (14) is equivalent to (3) when $n=0$.

In order to better understand the behavior of a PSL circuit, it is important to specialize the above equations for the case of a cisoidal excitation $x_{\text {in }}(t)=\left(X_{0} / 2 \pi\right) \exp (j \Omega t)$. The frequency-domain response $Y_{\text {out }}(\omega)$ of the system is obtained by substituting the frequency-domain description of the input signal $X_{\text {in }}(\omega)=X_{0} \delta(\omega-\Omega)$ into (14), leading to

$$
Y_{\text {out }}(\omega)=\sum_{n=-\infty}^{+\infty} H_{n}(\Omega) X_{0} \delta\left(\omega-\Omega-n \omega_{c}\right) .
$$


The above equation, that is the Fourier transform of a signal described by its Fourier series, can be rewritten in time-domain by means of a complex sum of trigonometric functions with angular frequencies $\Omega+n \omega_{c}$ :

$$
y_{\text {out }}(t)=\frac{1}{2 \pi} \sum_{n=-\infty}^{+\infty} H_{n}(\Omega) X_{0} \exp \left(j\left(\Omega+n \omega_{c}\right) t\right) .
$$

From the previous results it is clear that PSL circuits are completely described in both frequency- and time-domains by the aliasing transfer functions $H_{n}(\Omega)$ (see $[5,11]$ for a more general definition and additional details). It is relevant to remark that the complexity of the analytical solution of a PSL circuit is reasonable and affordable for the case or resistive switched circuits only (as the example of Figure 1 with the capacitor replaced by a short-circuit). In the more general case of dynamical circuits, however, the complexity increases and numerical methods or specialized techniques are needed to compute the solution of the system (e.g., see [4-6]).

In order to overcome the above limitations, the next two sections develop an alternative and effective approach to circuit analysis. A generic circuit is seen as the interconnection of linear time-invariant elements (as resistors, capacitors, inductors, ...) and PSL resistive elements (as the twoterminal element of Figure 2). Thereby, the circuit is solved by suitably reinterpreting the characteristics of the circuit elements within a new framework involving augmented voltage and current variables. The proposed solution allows solving any switching dynamical circuit that can be decomposed into the interconnection of the above two classes of elements.

\section{PSL Resistive Elements}

In this section, the constitutive relation of PSL resistive circuit elements and their equivalent characteristics expressed in terms of augmented time-invariant relations are derived. For conciseness, the discussion and the proposed procedure are based on the two-terminal switching element of Figure 2. The method, however, is general and can be readily applied to the multiport case. The above extension is briefly outlined hereafter in this section and is applied to solve an example problem involving a two-port PSL resistive block in Section 7.

5.1. Constitutive Relations. In the scheme of Figure 2, the PSL element is driven by an ideal voltage source to allow the computation of its generalized admittance representation, that is, the constitutive relation involving the port voltage $v(t)$ and the current $i(t)$ as the input and output variables, respectively. The resistive switching element is the same of Figure 1 where the switch $S$ is characterized by a periodic switching activity between the open and the close positions with a characteristic period $T$. The switch is assumed to be closed in the first half part of the period (e.g., $t \in[0, T / 2]$ ) and open in the remaining part. A cisoidal signal $e(t)=$ $\left(E_{0} / 2 \pi\right) \exp (j \Omega t)$ is chosen as the voltage excitation in this example.

Since the switching block does not include dynamical elements, the port current $i(t)$ can be alternatively interpreted as the juxtaposition in time of the current response computed from the analysis of the circuit of Figure 2 at each operating state of the switch (i.e., open and close). Hence, the current $i(t)$ writes

$$
\begin{aligned}
i(t)=\frac{E_{0}}{2 \pi} \exp (j \Omega t) \cdot & {\left[G_{1} \sum_{n=-\infty}^{+\infty} \Pi_{T / 2}(t-n T)\right.} \\
& \left.+G_{2} \sum_{n=-\infty}^{+\infty} \Pi_{T / 2}\left(t-n T-\frac{T}{2}\right)\right],
\end{aligned}
$$

where $G_{1}=2 / R$ and $G_{2}=1 / R$ are the equivalent conductances of the PSL two-terminal element when the switch is close or open, respectively, and $\Pi_{\Delta}\left(t-t_{0}\right)$ is the window function defined by

$$
\Pi_{\Delta}\left(t-t_{0}\right)= \begin{cases}1 & t_{0} \leq t \leq \Delta+t_{0} \\ 0 & \text { otherwise }\end{cases}
$$

The direct application of the Fourier transform to (17) leads, via a relatively long but straightforward manipulation, to

$$
\begin{gathered}
I(\omega)=\sum_{n=-\infty}^{+\infty} f_{c} c_{n}\left(G_{1}+G_{2} \exp \left(j n \omega_{c} \frac{T}{2}\right)\right) \\
\cdot E_{0} \delta\left(\omega-\Omega-n \omega_{c}\right),
\end{gathered}
$$

where $f_{c}=1 / T$ and $c_{n}$ are the coefficients of the Fourier series expansion of the periodic function $w(t)=\sum_{n=-\infty}^{+\infty} \Pi_{T / 2}(t-$ $n T)$ :

$$
\begin{aligned}
c_{n} & =\int_{0}^{T / 2} 1 \exp \left(-j n \omega_{c} t\right) d t \\
& =\frac{1-\exp \left(-j n \omega_{c} T / 2\right)}{j n \omega_{c}} .
\end{aligned}
$$

It is important to notice that (19) is equivalent to the general equation (14), where a single-frequency excitation $E(\omega)=E_{0} \delta(\omega-\Omega)$ is considered $(E(\omega)$ being the Fourier transform of the input cisoidal signal). Equations (14) and (19) allow defining the aliasing transfer functions as

$$
Y_{n}=f_{c} c_{n}\left(G_{1}+G_{2} \exp \left(j n \omega_{c} \frac{T}{2}\right)\right) .
$$

In this example, the functions $H_{n}=Y_{n}$ do not depend on the variable $\Omega$ since the circuit in each operating state is resistive and its solution involves instantaneous relations only. According to (13), the generalized bifrequency admittance can be written as

$$
Y(\omega, \Omega)=2 \pi \sum_{n=-\infty}^{+\infty} Y_{n} \cdot \delta\left(\omega-\Omega-n \omega_{c}\right)
$$

The readers should refer to the appendix that collects additional details on the behavior of the example circuit of Figure 2 with the aim of further stressing the key features of PSL circuits.

It is relevant to notice that the proposed procedure can be applied to multiport resistive elements as well, provided 


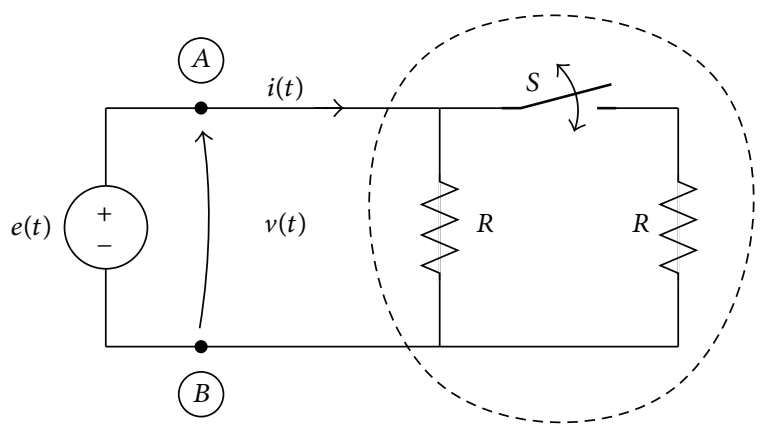

(a)

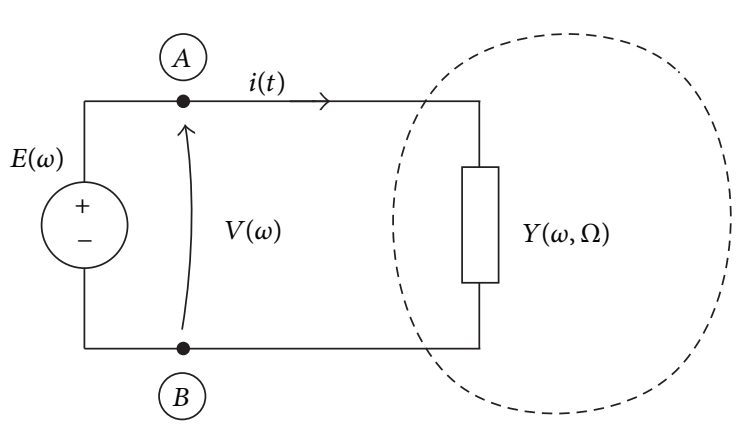

(b)

FIGURE 2: (a) Example circuit consisting of the interconnection of an ideal voltage source and of a switching resistive PSL block. (b) Circuit with the PSL block described by means of its generalized bifrequency admittance $Y(\omega, \Omega)$.

that their port admittance characteristic can be computed as for the example two-terminal element of Figure 2. In practice, voltage sources are applied to the different ports of the multiport and the current responses are interpreted as the juxtaposition in time of the port current responses computed for the different operating states of the switches, leading to matrix relations similar to (19).

5.2. Augmented Characteristics. Equation (19) suggests that the frequency-domain steady-state response of a PSL circuit with independent voltage or current sources defined by cisoidal excitations with angular frequency $\Omega=\omega_{0}$ can be suitably expressed in terms of an infinite sum of delta functions [11]. For practical reasons, this infinite series is then truncated. For example, for the case of the circuit of Figure 2,

$$
\begin{aligned}
I(\omega) & =\sum_{n=-\infty}^{+\infty} I_{n} \delta\left(\omega-n \omega_{c}-\omega_{0}\right) \\
& \approx \sum_{n=-N}^{+N} I_{n} \delta\left(\omega-n \omega_{c}-\omega_{0}\right),
\end{aligned}
$$

where $I_{n}$ are the Fourier coefficients of the $n$th harmonic of the current through the PSL element and $N$ is the total number of (both positive and negative) harmonics considered, $(2 N+1)$ being the total number of terms defining the expansion (23). A similar relation holds for the voltage $V(\omega)$.

The previous interpretation of the electrical variables along with (7) (with $Y_{\text {out }}=I$ and $X_{\text {in }}=V$ ) and (21) leads to the following generalized Ohm's law describing the PSL resistive block:

$$
\begin{aligned}
I(\omega) & =\frac{1}{2 \pi} \int_{-\infty}^{+\infty} Y(\omega, \Omega) V(\Omega) d \Omega \\
& \approx \sum_{k=-N}^{N} \sum_{m=-2 N}^{2 N} V_{k} Y_{m} \delta\left(\omega-(m+k) \omega_{c}-\omega_{0}\right),
\end{aligned}
$$

for $|m+k| \leq N$.

It is important to notice that the periodic behavior of the system and the convenient interpretation of voltages and currents in terms of series expansions allow replacing the integral operator with the products of two sums. From the above equation it is also clear that voltage and current variables are defined by truncated Fourier expansions, which take into account $2 N+1$ harmonics, from $-N \omega_{c}+\omega_{0}$ to $+N \omega_{c}+\omega_{0}$.

Equation (24), along with the representation of the current $I(\omega)$ by means of (23), can be represented via the following augmented characteristic equation of the twoterminal PSL element in matrix form:

$$
\left[\begin{array}{c}
I_{-N} \\
\vdots \\
I_{0} \\
\vdots \\
I_{N}
\end{array}\right]=\left[\begin{array}{ccccc}
Y_{0} & Y_{-1} & \cdots & \cdots & Y_{-2 N} \\
Y_{1} & Y_{0} & \cdots & \cdots & \vdots \\
\vdots & \vdots & \ddots & \vdots & \vdots \\
\vdots & \vdots & \vdots & \ddots & Y_{-1} \\
Y_{2 N} & \cdots & \cdots & Y_{1} & Y_{0}
\end{array}\right]\left[\begin{array}{c}
V_{-N} \\
\vdots \\
V_{0} \\
\vdots \\
V_{N}
\end{array}\right]
$$

where the voltage and current variables $V(\omega)$ and $I(\omega)$ are replaced by the vectors $\mathbf{V}=\left[V_{-N}, \ldots, V_{0}, \ldots, V_{N}\right]^{T}$ and $\mathbf{I}=$ $\left[I_{-N}, \ldots, I_{0}, \ldots, I_{N}\right]^{T}$. The above vectors, that have dimension $(2 N+1) \times 1$, are filled in by the harmonic coefficients defining the steady-state response of the corresponding variables and play the role of the new port variables of a possibly augmented circuit element. Clearly, this is the same underlying interpretation of the so-called harmonic balance that has been used along with other methods for the solution of PSL circuits in [19-22]. In the latter papers, however, the proposed simulation technique involves more complex and cumbersome computations. In our proposal, instead, the complexity of the circuit solution is dramatically reduced by the interpretation of the characteristics of PSL blocks according to (25).

Equation (25), that turns out to be a linear time-invariant representation of the PSL element, can be written in a more compact form as

$$
\mathbf{I}=\mathbf{Y V}
$$

where $\mathbf{Y}$ is the $(2 N+1) \times(2 N+1)$ matrix of $(25)$.

For conciseness, the above procedure has been detailed for the case of a two-terminal element only. Its extension 


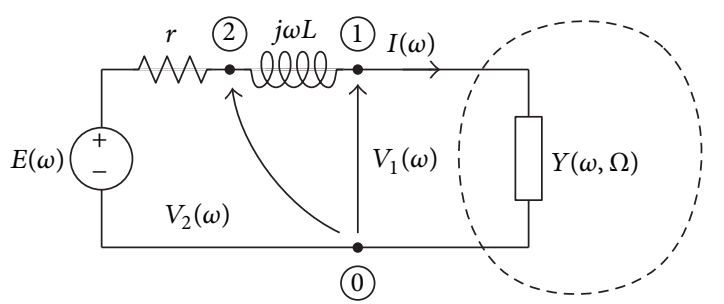

Figure 3: Example test circuit used for illustrating the proposed MNA formulation. The values defining the LTI series elements, that are characterized by their frequency-domain impedances, are $r=$ $10 \Omega, L=1 \mathrm{mH}$. The PSL block highlighted by the dashed frame is the two-terminal PSL resistive block of Figure 2, where $R=100 \Omega$ and the switch $S$ has a periodic switching activity at frequency $f_{c}=$ $10 \mathrm{kHz}$. The independent DC voltage source $e(t)=10 \mathrm{~V}$ is defined in frequency-domain as $E(\omega)=10 / 2 \pi \delta(\omega) \mathrm{V}$.

to the more general multiport case is straightforward and requires to follow the same steps above.

\section{Circuit Solution via Augmented MNA}

This section adopts the classical MNA approach for circuit analysis to the case of PSL circuits. For the sake of illustration, the derivation is based on the example circuit of Figure 3, where a nonideal voltage source with inductive series impedance is connected to the switching resistive block of Figure 2. In the schematic of Figure 3, the PSL block is represented by its generalized bifrequency admittance $Y(\omega, \Omega)$ defined by means of (22) and the ideal voltage source $e(t)$ is defined by the cisoidal excitation $e(t)=$ $\left(E_{0} / 2 \pi\right) \exp \left(j \omega_{0} t\right)$.

The discussion starts with the well-known stamp arising from the analysis of the schematic of Figure 3 where a simple admittance $Y$ (e.g., $Y(\omega)=j \omega C$ ) replaces the PSL block. In this case, the MNA equation in matrix form writes

$$
\left[\begin{array}{cc|c}
Y(\omega) & 0 & -1 \\
0 & g & 1 \\
\hline-1 & 1 & -j \omega L
\end{array}\right]\left[\begin{array}{c}
V_{1}(\omega) \\
V_{2}(\omega) \\
I(\omega)
\end{array}\right]=\left[\begin{array}{c}
0 \\
g E(\omega) \\
0
\end{array}\right] .
$$

When the impedance $Y(\omega)$ is substituted by $Y(\omega, \Omega)$, the unknowns, that is, $V_{1}, V_{2}$, and $I$, need to be replaced by the corresponding vectors $\mathbf{V}_{1}, \mathbf{V}_{2}$, and I collecting the coefficients of the harmonic series expansion of the nodal unknowns.

The augmented characteristic of the PSL two-terminal element defined by (25) and (26) and the companion relations that can be readily obtained for the classical LTI elements allow replacing the original MNA equation with

$$
\left[\begin{array}{cc|c}
\mathbf{Y} & \mathbf{0} & -\mathbf{1} \\
\mathbf{0} & g \mathbf{1} & \mathbf{1} \\
\hline-\mathbf{1} & \mathbf{1} & -\mathbf{Z}_{L}
\end{array}\right]\left[\begin{array}{c}
\mathbf{V}_{1} \\
\mathbf{V}_{2} \\
\mathbf{I}
\end{array}\right]=\left[\begin{array}{c}
\mathbf{0} \\
g \mathbf{E} \\
\mathbf{0}
\end{array}\right]
$$

where all the entries of the matrix are submatrices with dimension $(2 N+1) \times(2 N+1)$, where $\mathbf{1}=\operatorname{diag}(1), \mathbf{0}=$ $\operatorname{diag}(0)$, and $\mathbf{Z}_{L}=\operatorname{diag}\left(j\left[\ldots,\left(\omega_{0}-\omega_{c}\right), \omega_{0},\left(\omega_{0}+\omega_{c}\right), \ldots\right] L\right)$. On the right hand side of the MNA equation, the excitation term is composed of vectors of dimension $(2 N+1) \times 1$ defined as $\mathbf{0}=[0, \ldots, 0]^{T}$ and $\mathbf{E}=\left[0, \ldots, E_{0}, \ldots, 0\right]^{T}$.

Clearly, (28) turns out to be $(2 N+1)$ times larger than the corresponding MNA equation of a circuit with the PSL element replaced by a linear time-invariant impedance. However, the proposed extended matrix, that belongs to the same class of (27), can be readily solved via simple linear inversion. What is more important, the steady-state response of the PSL circuit can be computed by reinterpreting the coefficients of the new voltage and current unknowns by means of (23). Equation (29) defines the unwrapped and complete extended MNA stamp for the example circuit of Figure 3 with $N=1$.

In sum, the proposed simulation method is valid for PSL circuits with both constant sources (a constant is seen as a cisoidal signal with null angular frequency) and any arbitrary periodic excitation, provided that the latter signal is expended in Fourier series. Also, the solution of the augmented MNA equation like (29) provides the direct computation of the steady-state response of the circuit.

For the sake of illustration, the method has been derived for the case of a time-varying circuit with one PSL resistive two-terminal element only. The method, however, is general and can be suitably applied to arbitrary circuits with one or more multiport resistive PSL elements, provided that the switching elements can be described by means of an admittance representation. It is also useful to remark that the proposed method is still valid if the resistive switching element is described by its impedance representation. The same procedure suggested above for the generation of the augmented characteristic (26) of the example two-terminal element of Figure 1 can be followed. As an example, Section 7 includes the results obtained by considering a circuit with a two-port switching resistive element and briefly outlines the procedure for the computation of its augmented characteristic. The solution of the proposed augmented MNA problem introduces benefits in terms of robustness and efficiency with respect to the classical ODE piecewise approach in timedomain.

\section{Numerical Results}

This section collects the numerical results obtained by applying the proposed method to the solution of the two example circuits. The responses of the circuits computed by means of MATLAB and the standard ordinary differential equation (ODE) integration routines are assumed as the reference curves in this study. The reference curves are compared with the solution obtained by means of the linear inversion of the augmented matrix equation like (29).

The first validation deals with the same example circuit of Figure 3. For this test case, Figures 4 and 5 show both the time- and frequency-domain steady-state responses of the current $i(t)$ and voltage $v_{1}(t)$, respectively, thus highlighting the good accuracy of the proposed method in reproducing the reference responses. The time-domain comparison is carried out by considering the series approximation of the 

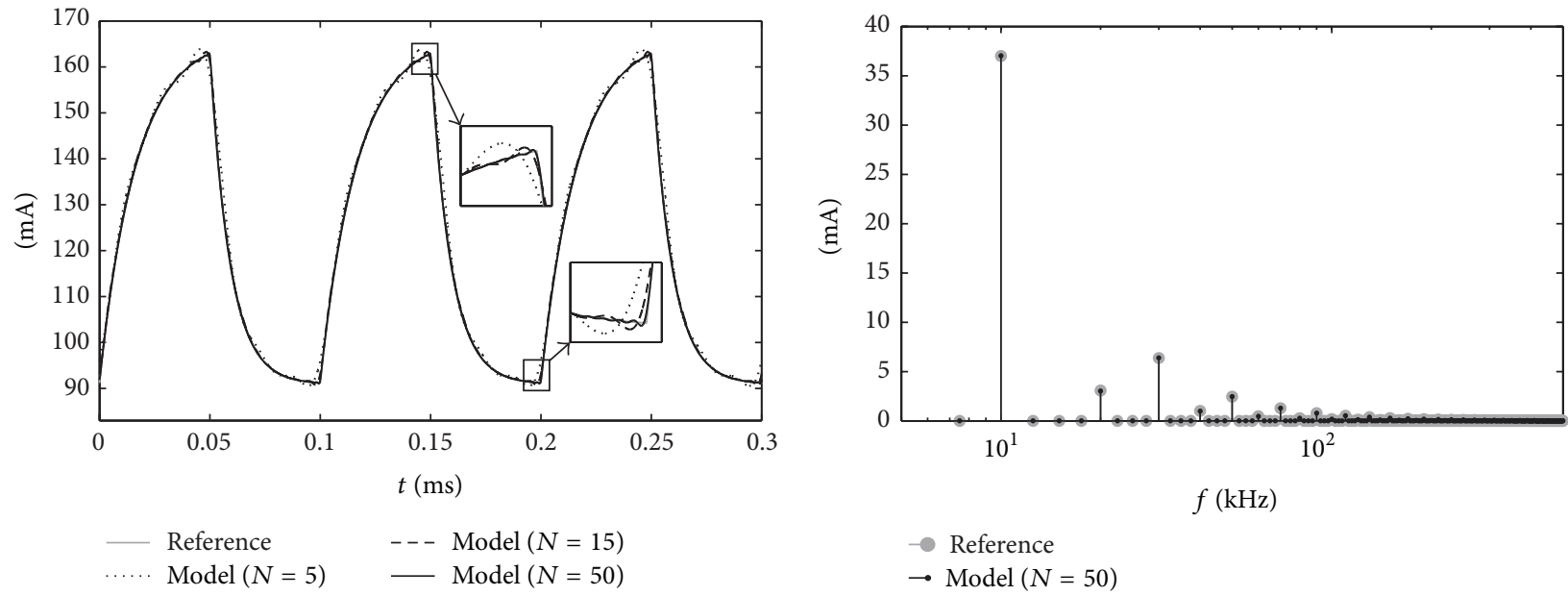

FIGURE 4: Time-domain (steady-state) responses of the current $i(t)$ for the circuit of Figure 3 and its corresponding frequency-domain spectrum. The reference time-domain response (computed by means of the ODE-based routines in MATLAB) is compared with the solutions arising from (29) and different values of the order of expansion $N$.
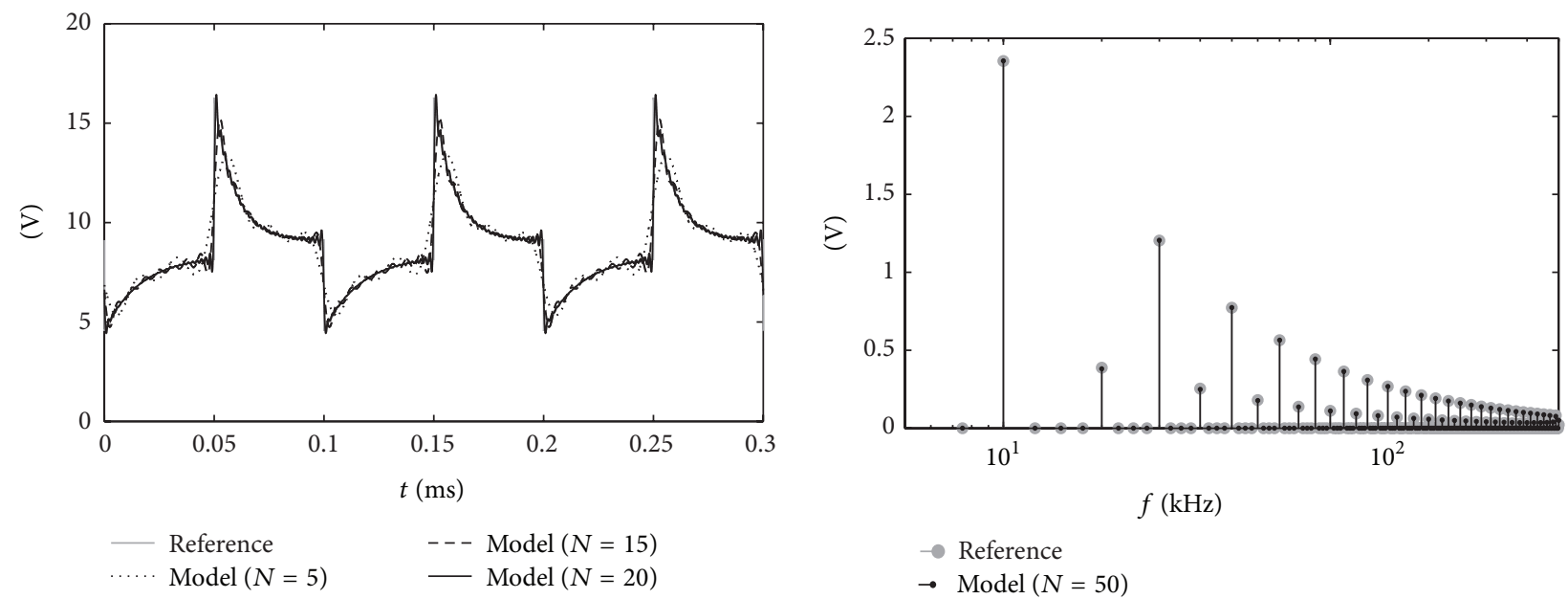

FIGURE 5: Time-domain (steady-state) responses of the voltage $v_{2}(t)$ for the circuit of Figure 3 and its corresponding frequency-domain spectrum. The reference time-domain response (computed by means of the ODE-based routines in MATLAB) is compared with the solutions arising from (29) and different values of the order of expansion $N$.

unknowns with an increasing number of the expansion orders $N$. For plot readability, a maximum order of 50 has been considered since a larger order generates responses overlapping the reference curve very well and does not allow appreciating the differences among the approximated responses:

$$
\left[\begin{array}{cccccc|ccc}
Y_{0} & Y_{-1} & Y_{-2} & 0 & 0 & 0 & -1 & 0 & 0 \\
Y_{1} & Y_{0} & Y_{-1} & 0 & 0 & 0 & 0 & -1 & 0 \\
Y_{2} & Y_{1} & Y_{0} & 0 & 0 & 0 & 0 & 0 & -1 \\
0 & 0 & 0 & g & 0 & 0 & 1 & 0 & 0 \\
0 & 0 & 0 & 0 & g & 0 & 0 & 1 & 0 \\
0 & 0 & 0 & 0 & 0 & g & 0 & 0 & 1 \\
\hline-1 & 0 & 0 & 1 & 0 & 0 & -j\left(\omega_{0}-\omega_{c}\right) L & 0 & 0 \\
0 & -1 & 0 & 0 & 1 & 0 & 0 & -j\left(\omega_{0}\right) L & 0 \\
0 & 0 & -1 & 0 & 0 & 1 & 0 & 0 & -j\left(\omega_{0}+\omega_{c}\right) L
\end{array}\right]\left[\begin{array}{c}
V_{1,-1} \\
V_{1,0} \\
V_{1,1} \\
V_{2,-1} \\
V_{2,0} \\
V_{2,1} \\
I_{-1} \\
I_{0} \\
I_{1}
\end{array}\right]=\left[\begin{array}{c}
0 \\
0 \\
0 \\
0 \\
g E_{0} \\
0 \\
0 \\
0
\end{array}\right] .
$$


TABLE 1: CPU-time required by the simulation of the circuit of Figure 3 and accuracy of the computed current and voltage responses $i(t)$ and $v_{1}(t)$, for an increasing number of terms $N$ defining their truncated expansions (e.g., see (23)).

\begin{tabular}{lcccccccc}
\hline Method & ODE $t_{\text {step }}=1 \mathrm{~ns}$ & \multicolumn{5}{c}{ Extended MNA } \\
& $t_{\max }=500 \mu \mathrm{s}$ & $N=1$ & $N=5$ & $N=15$ & $N=50$ & $N=75$ & $N=100$ & $N=150$ \\
\hline CPU-time & $18.7 \mathrm{~s}$ & $0.052 \mathrm{~s}$ & $0.059 \mathrm{~s}$ & $0.079 \mathrm{~s}$ & $0.15 \mathrm{~s}$ & $0.17 \mathrm{~s}$ & $0.23 \mathrm{~s}$ & $0.46 \mathrm{~s}$ \\
Relative error $i(t)$ & Reference & $4.7 \%$ & $1.3 \%$ & $0.4 \%$ & $0.13 \%$ & $0.09 \%$ & $0.06 \%$ & $0.04 \%$ \\
Relative error $v_{1}(t)$ & Reference & $15 \%$ & $9.5 \%$ & $5.9 \%$ & $3.4 \%$ & $2.7 \%$ & $2.42 \%$ & $1.98 \%$ \\
\hline
\end{tabular}

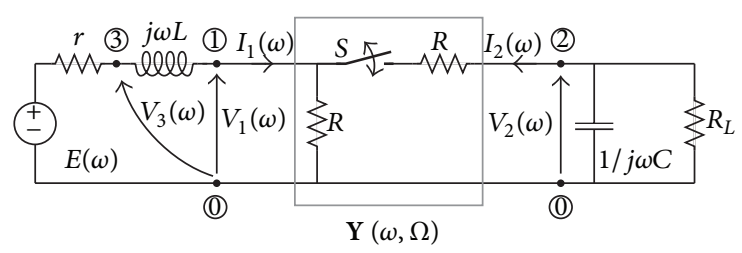

FIGURE 6: Example circuit involving a two-port PSL resistive block and standard (both resistive and dynamical) LTI elements. The values of components are $r=10 \Omega, L=1 \mathrm{mH}, R=100 \Omega$, $R_{L}=100 \Omega$, and $C=1 \mu \mathrm{F}$. The switch operates as in the first example of Figure 3. The independent DC voltage source $e(t)=$ $10+2 \sin \left(\omega_{0} t\right) \mathrm{V}$ is defined in frequency-domain as $E(\omega)=$ $10 /(2 \pi) \delta(\omega)+1 / j \pi\left(\delta\left(\omega-\omega_{0}\right)-\delta\left(\omega+\omega_{0}\right)\right) \mathrm{V}$, where $\omega_{0}=2 \pi f_{0}$ and $f_{0}=40 \mathrm{kHz}$.

In order to provide a better quantitative comparison, Table 1 collects the main figures on the accuracy and efficiency of the ODE-based and of the proposed MNAbased methods implemented in MALAB environment. The accuracy is assessed by computing the relative mean square error between the reference and the predicted responses. Also, the overhead of the proposed method in computing the aliasing admittances $Y_{n}$ is included in the total CPU-time. The numbers in the table confirm the strengths of the proposed approach that has a number of advantages over alternative state-of-the-art methods. Mainly, it allows computing the solution of the nodal variables of a periodically switched circuit in both frequency- and time-domains by means of the solution of a single linear problem. The coefficients in the unknown vector of (29) are the numbers multiplying either the complex trigonometric functions defining the time-domain responses or the deltas defining the different terms of the corresponding frequency-domain spectra.

As a second and more realistic test case, the circuit of Figure 6 is considered. It is composed of a two-port PSL resistive block (highlighted by means of a gray frame) and standard linear time-invariant two-terminal elements. The topology of this second example is similar to the ones occurring in a number of applications (e.g., the DC-DC boost converter is defined by a similar circuit with a different PSL block involving two switching components, i.e., the diode and the MOS transistor). As already done in Section 5 for the two-terminal PSL element of Figure 3, the characteristic of the two-port resistive PSL block needs to be suitably replaced by an approximated characteristic involving the expansion of its port voltages and currents (e.g., see (24) and
(26)). Specifically, the integral equations governing the port behavior of the PSL block,

$$
\begin{aligned}
I_{1}(\omega)= & \frac{1}{2 \pi} \int_{-\infty}^{+\infty} Y_{11}(\omega, \Omega) V_{1}(\Omega) d \Omega \\
& +\frac{1}{2 \pi} \int_{-\infty}^{+\infty} Y_{12}(\omega, \Omega) V_{2}(\Omega) d \Omega \\
I_{2}(\omega)= & \frac{1}{2 \pi} \int_{-\infty}^{+\infty} Y_{21}(\omega, \Omega) V_{1}(\Omega) d \Omega \\
& +\frac{1}{2 \pi} \int_{-\infty}^{+\infty} Y_{22}(\omega, \Omega) V_{2}(\Omega) d \Omega,
\end{aligned}
$$

can be suitably replaced, via the same procedure of Section 5, by

$$
\left[\begin{array}{l}
\mathbf{I}_{1} \\
\mathbf{I}_{2}
\end{array}\right]=\left[\begin{array}{ll}
\mathbf{Y}_{11} & \mathbf{Y}_{12} \\
\mathbf{Y}_{21} & \mathbf{Y}_{22}
\end{array}\right]=\left[\begin{array}{l}
\mathbf{V}_{1} \\
\mathbf{V}_{2}
\end{array}\right],
$$

where the interpretation of the variables and matrices is the same as the one introduced in the previous section. From the above equation, it is clear that the PSL block is now suitable to be plugged into the augmented MNA equation describing the circuit of Figure 6, leading to

$$
\left[\begin{array}{ccc|c}
\mathbf{Y}_{11} & \mathbf{Y}_{12} & \mathbf{0} & -\mathbf{1} \\
\mathbf{Y}_{21} & \mathbf{Y}_{22}+\mathbf{Y}_{R C} & \mathbf{0} & \mathbf{0} \\
\mathbf{0} & \mathbf{0} & g \mathbf{1} & \mathbf{1} \\
\hline-\mathbf{1} & \mathbf{0} & \mathbf{1} & -\mathbf{Z}_{L}
\end{array}\right]\left[\begin{array}{l}
\mathbf{V}_{1} \\
\mathbf{V}_{2} \\
\mathbf{V}_{3} \\
\mathbf{I}_{1}
\end{array}\right]=\left[\begin{array}{c}
\mathbf{0} \\
\mathbf{0} \\
g \mathbf{E} \\
\mathbf{0}
\end{array}\right]
$$

where $\mathbf{Y}_{R C}=\operatorname{diag}\left(j\left[\ldots,\left(\omega_{0}-\omega_{c}\right), \omega_{0},\left(\omega_{0}+\omega_{c}\right), \ldots\right] C\right)+$ $\operatorname{diag}\left(G_{L}\right)$ is a submatrix with dimensions $(2 N+1) \times(2 N+1)$ and $G_{L}=1 / R_{L}$. The remaining entries of the matrix and the nodal unknowns are the same already defined in (29).

Figure 7 shows the reference and the predicted responses of the voltage $v_{2}(t)$ and the current $i_{1}(t)$ of this second test circuit. The curves in the figure highlight that the proposed method produces the steady-state responses of a PSL circuit and does not include the initial transient observed in the reference response of the circuit calculated via an ODEbased solver with null initial conditions of the dynamical components. As far as the performance of the proposed method is concerned, the trend given by the numbers of Table 1 is also confirmed for this second example (see Table 2 for detailed information on the accuracy and performance of the method for an increasing number of the expansion orders $N)$. This second example allows claiming the generality of the proposed approach that can be applied to circuits with 


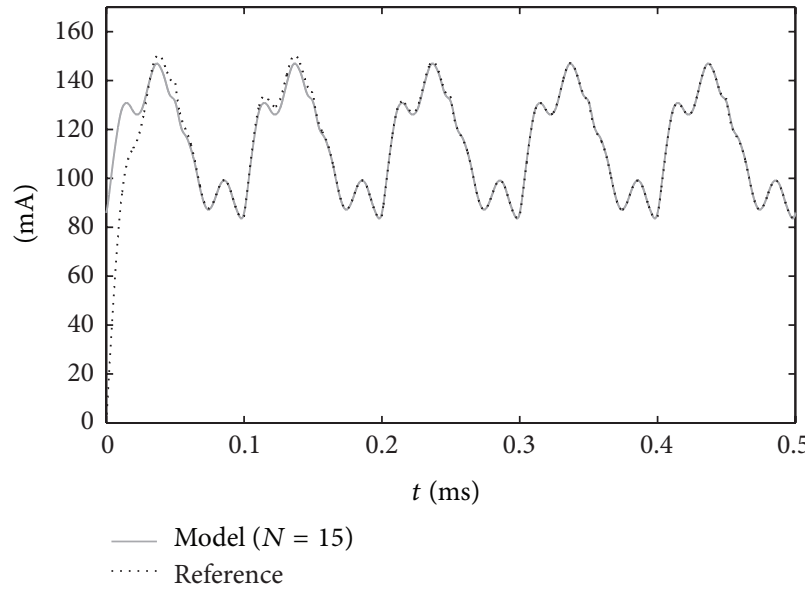

(a) Current $i_{1}(t)$

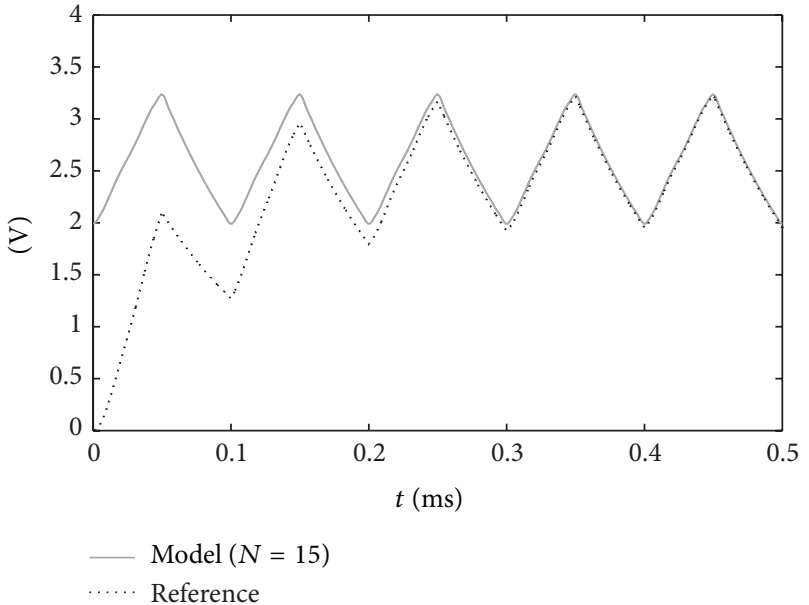

(b) Voltage $v_{2}(t)$

FIGURE 7: Time-domain voltage and current responses of the circuit of Figure 6 . The reference curves are compared with the solutions arising from (32) and the order of expansion $N=15$.

TABLE 2: CPU-time required by the simulation of the circuit of Figure 6 and accuracy of the computed current and voltage responses $i_{1}(t)$ and $v_{2}(t)$, for an increasing number of terms $N$ defining their truncated expansions (e.g., see (23)).

\begin{tabular}{|c|c|c|c|c|c|c|c|c|}
\hline \multirow{2}{*}{ Method } & \multirow{2}{*}{$\begin{array}{l}\mathrm{ODE} t_{\text {step }}=1 \mathrm{~ns} \\
t_{\max }=500 \mu \mathrm{s}\end{array}$} & \multicolumn{7}{|c|}{ Extended MNA } \\
\hline & & $N=1$ & $N=5$ & $N=15$ & $N=50$ & $N=75$ & $N=100$ & $N=150$ \\
\hline CPU-time & $34.5 \mathrm{~s}$ & $0.029 \mathrm{~s}$ & $0.051 \mathrm{~s}$ & $0.076 \mathrm{~s}$ & $0.19 \mathrm{~s}$ & $0.24 \mathrm{~s}$ & $0.445 \mathrm{~s}$ & $1.1 \mathrm{~s}$ \\
\hline Relative error $i(t)$ & Reference & $3.3 \%$ & $0.9 \%$ & $0.3 \%$ & $0.08 \%$ & $0.05 \%$ & $0.04 \%$ & $0.02 \%$ \\
\hline Relative error $v_{1}(t)$ & Reference & $4.9 \%$ & $1.7 \%$ & $0.6 \%$ & $0.2 \%$ & $0.14 \%$ & $0.1 \%$ & $0.07 \%$ \\
\hline
\end{tabular}

multiterminal PSL blocks without modifying the basic rules introduced in the previous section for the generation of the augmented MNA equation.

\section{Conclusions}

This paper addressed the simulation of the steady-state behavior of an important class of time-varying circuits consisting of linear time-invariant (possibly dynamical) circuit elements, periodically switched linear resistive multiport elements, and ideal sources defined by periodic excitations. The proposed solution extends the results of previously published papers in this field and is based on the generation of an augmented time-invariant MNA equation governing the circuit behavior. The new nodal unknowns in the advocated MNA equation correspond to the harmonic coefficients defining the expansions of the unknown voltage and current variables. A topological approach is considered, with emphasis on an intuitive physical based interpretation of the PSL elements in the network. The proposed solution is proven to offer a modular approach to circuit analysis, leading to accurate results with good simulation speed-ups. Also, it is sufficiently general to handle a wide class of circuit topologies occurring in real application problems. The feasibility and strengths of the method are demonstrated on simple analytical examples and two validation test circuits.

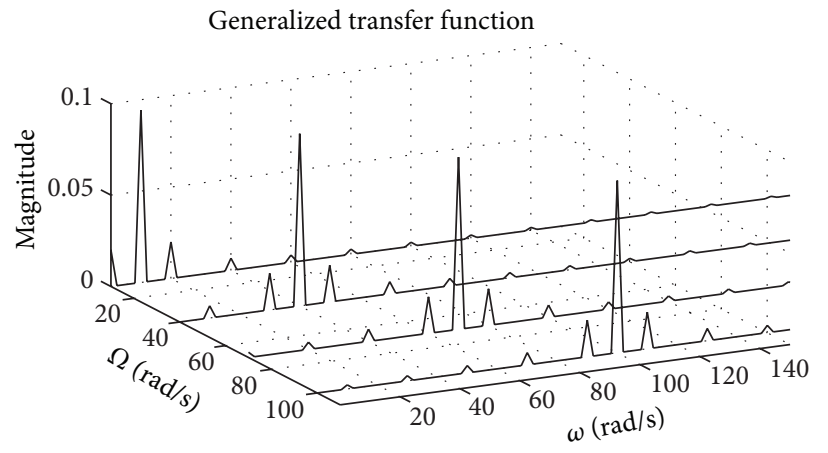

FIGURE 8: Bifrequency generalized admittance $H(\omega, \Omega)$ associated with the example circuit of Figure 2.

\section{Appendix}

This appendix collects some useful remarks on the general behavior of a PSL circuit to highlight its features and to provide a graphical interpretation of the operators involved in the circuit analysis. The discussion is based on the circuit of Figure 2.

As an example, Figure 8 shows the magnitude of $Y(\omega, \Omega)$ defined by (22) and computed for $R=100 \Omega$ and $f_{c}=$ $10 \mathrm{kHz}$. To improve the readability of the plot, the $\Omega$-axis is discretized and four samples are considered only. This 


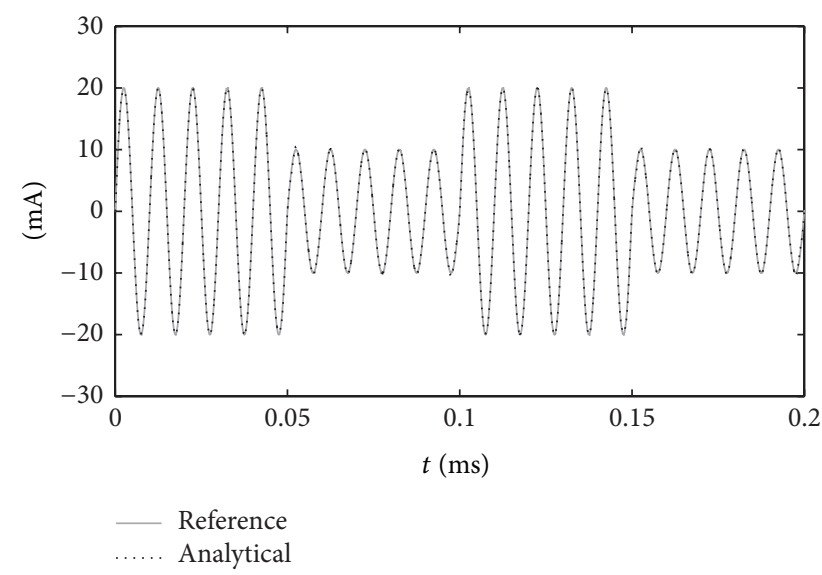

(a)

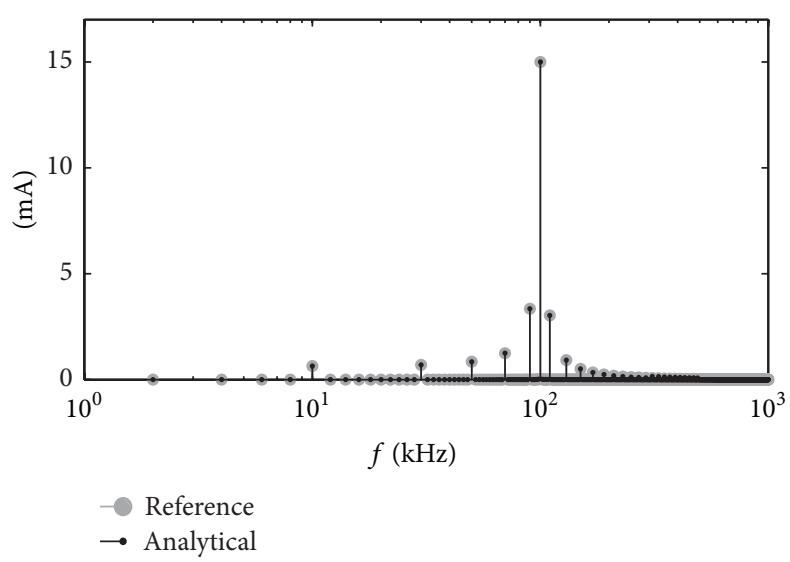

(b)

FIgURE 9: Time-domain response of the current $i(t)$ (a) of the example circuit in Figure 2 and its corresponding spectrum $I(f)(b)$.

figure allows getting a practical interpretation of the transfer function of a time-varying system. Due to the term $\delta(\omega-\Omega-$ $\left.n \omega_{c}\right)$ in (13), the cut of the two-dimensional surface $Y(\omega, \Omega)$ for a fixed $\Omega$ value turns out to be given by the superposition of delta functions occurring at frequencies $\Omega+n \omega_{c}(n=$ $\ldots,-2,-1,0,1, \ldots)$. Clearly, the position of such deltas on the $\omega$-axis are shifted to the right (or to the left) when the values of the input frequencies $\Omega$ increase (or decrease).

Figure 9 shows the time-domain current response $i(t)$ of the circuit of Figure 2 to a sinusoidal excitation $e(t)=$ $\sin \left(2 \pi f_{0} t\right), f_{0}=100 \mathrm{kHz}$, and its corresponding frequencydomain spectrum. The above figure highlights that the steady-state response of a periodically time-varying system contains an infinite number of components at frequencies $\pm \omega_{0}+n \omega_{c}$.

The example discussed in this appendix clearly illustrates that a linear time-varying circuit excited by a sinusoidal signal produces new harmonics even if the circuit does not contain active elements (i.e., the circuit of Figure 2 has an ideal voltage source connected to a passive two-terminal switching resistive element).

\section{Conflict of Interests}

The authors declare that there is no conflict of interests regarding the publication of this paper.

\section{References}

[1] L. A. Zadeh, "Frequency analysis of variable networks," Proceedings of the IRE, vol. 38, no. 3, pp. 291-299, 1950.

[2] L. A. Zadeh and C. A. Desoer, Linear System Theory: The State Space Approach, McGraw-Hill, New York, NY, USA, 1963.

[3] A. Papoulis, Signal Analysis, McGraw-Hill, Auckland, New Zealand, 1977.

[4] M.-L. Liou, "Exact analysis of linear circuits containing periodically operatd wwthes with applications," IEEE Transactions on Circuit Theory, vol. 19, no. 2, pp. 146-154, 1972.
[5] T. Strom and S. Signell, "Analysis of periodically switched linear circuits," IEEE Transactions on Circuits and Systems, vol. 24, no. 10, pp. 531-541, 1977.

[6] F. Yuan and A. Opal, Computer Methods for Analysis of MixedMode Switching Circuits, Kluwer Academic, New York, NY, USA, 2004.

[7] F. Yuan and A. Opal, "Sensitivity analysis of periodically switched linear circuits using an adjoint network technique," in Proceedings of the IEEE International Symposium on Circuits and Systems (ISCAS '99), pp. V-331-V-334, June 1999.

[8] J. A. Á. Martín, J. R. Melgoza, and J. J. R. Pasaye, "Exact steady state analysis in power converters using Floquet decomposition," in Proceedings of the North American Power Symposium (NAPS '11), pp. 1-7, Boston, Mass, USA, August 2011.

[9] J. Kovar, Z. Kolka, and D. Biolek, "Comparison of averaging and harmonic balance methods for switched DC-DC converters," in Proceedings of the 17th International Conference on Mixed Design of Integrated Circuits and Systems (MIXDES '10), pp. 402-407, Warsaw, Poland, June 2010.

[10] H. Behjati, L. Niu, A. Davoudi, and P. L. Chapman, "Alternative time-invariant multi-frequency modeling of PWM DCDC converters," IEEE Transactions on Circuits and Systems I: Regular Papers, vol. 60, no. 11, pp. 3069-3079, 2013.

[11] T. A. C. M. Claasen and W. F. G. Mecklenbrauker, "On stationary linear time-varying systems," IEEE Transactions on Circuits and Systems, vol. 29, no. 3, pp. 169-184, 1982.

[12] H. Sandberg, E. Mollerstedt, and Bernhardsson, "Frequencydomain analysis of linear time-periodic systems," IEEE Transactions on Automatic Control, vol. 50, no. 12, pp. 1971-1983, 2005.

[13] P. M. Mellacheruvu, S. M. Mahajan, C. L. Carnal, and J. J. Biernacki, "Application of fourier and laplace transform techniques for modeling an electric circuit having time-varying components," in Proceedings of the 38th Southeastern Symposium on System Theory (SSST '06), pp. 147-151, March 2006.

[14] J. Lerdworatawe and W. Namgoong, "Generalized linear periodic time-varying analysis for noise reduction in an active mixer," IEEE Journal of Solid-State Circuits, vol. 42, no. 6, pp. 1339-1351, 2007.

[15] M. L. Liou, Y.-L. Kuo, and C. F. Lee, "A tutorial on computeraided analysis of switched-capacitor circuits," Proceedings of the IEEE, vol. 17, no. 8, pp. 987-1005, 1983. 
[16] R. Trinchero, I. S. Stievano, and F. G. Canavero, "Steady-state analysis of switching power converters via augmented timeinvariant equivalents"', IEEE Transactions on Power Electronics, vol. 29, no. 11, pp. 5657-5661, 2014.

[17] H. Bishnoi, P. Mattavelli, R. Burgos, and D. Boroyevich, "EMI behavioral models of DC-fed three-phase motor drive systems," IEEE Transactions on Power Electronics, vol. 29, no. 9, pp. 46334645, 2014

[18] I. Cadirci, B. Saka, and Y. Eristiren, "Practical EMI-filter-design procedure for high-power high-frequency SMPS according to MIL-STD 461," IEE Proceedings-Electric Power Applications, vol. 152, no. 4, pp. 775-782, 2005.

[19] M. Okumura, H. Tanimoto, T. Itakura, and T. Sugawara, "Numerical noise analysis for nonlinear circuits with a periodic large signal excitation including cyclostationary noise sources," IEEE Transactions on Circuits and Systems I: Fundamental Theory and Applications, vol. 40, no. 9, pp. 581-590, 1993.

[20] P. Dobrovolny, G. Vandersteen, P. Wambacq, and S. Donnay, "Analysis and white-box modeling of weakly nonlinear timevarying circuits," in Proceedings of the Design, Automation and Test in Europe Conference and Exhibition, pp. 624-629, 2003.

[21] J. Roychowdhury, "Reduced-order modeling of time-varying systems," IEEE Transactions on Circuits and Systems II: Analog and Digital Signal Processing, vol. 46, no. 10, pp. 1273-1288, 1999.

[22] T. Mei, H. Thornquist, E. Keiter, and S. Hutchinson, "Structure preserving reduced-order modeling of linear periodic timevarying systems," in Proceedings of the IEEE/ACM International Conference on Computer-Aided Design (ICCAD '11), pp. 361366, San Jose, Calif, USA, November 2011. 

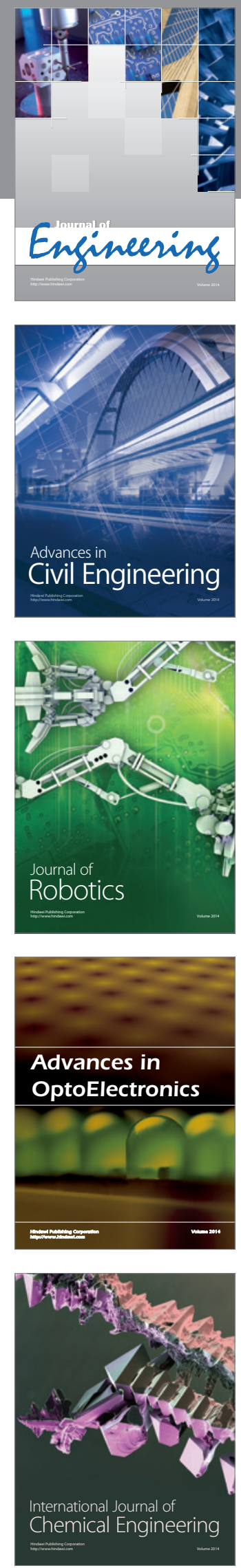

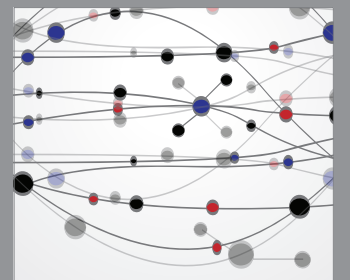

The Scientific World Journal
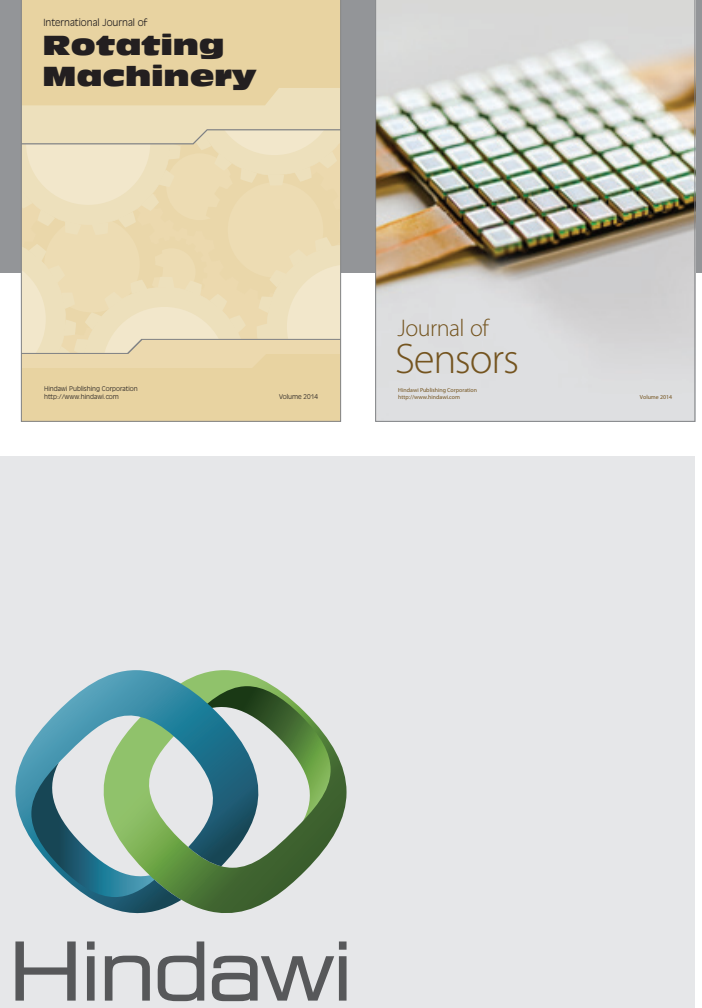

Submit your manuscripts at http://www.hindawi.com
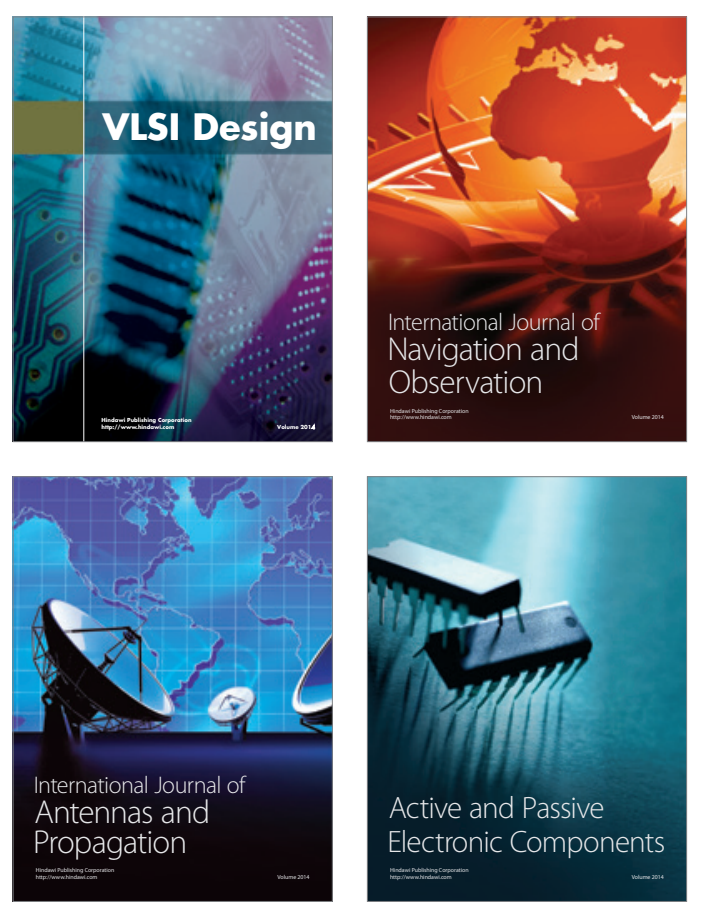
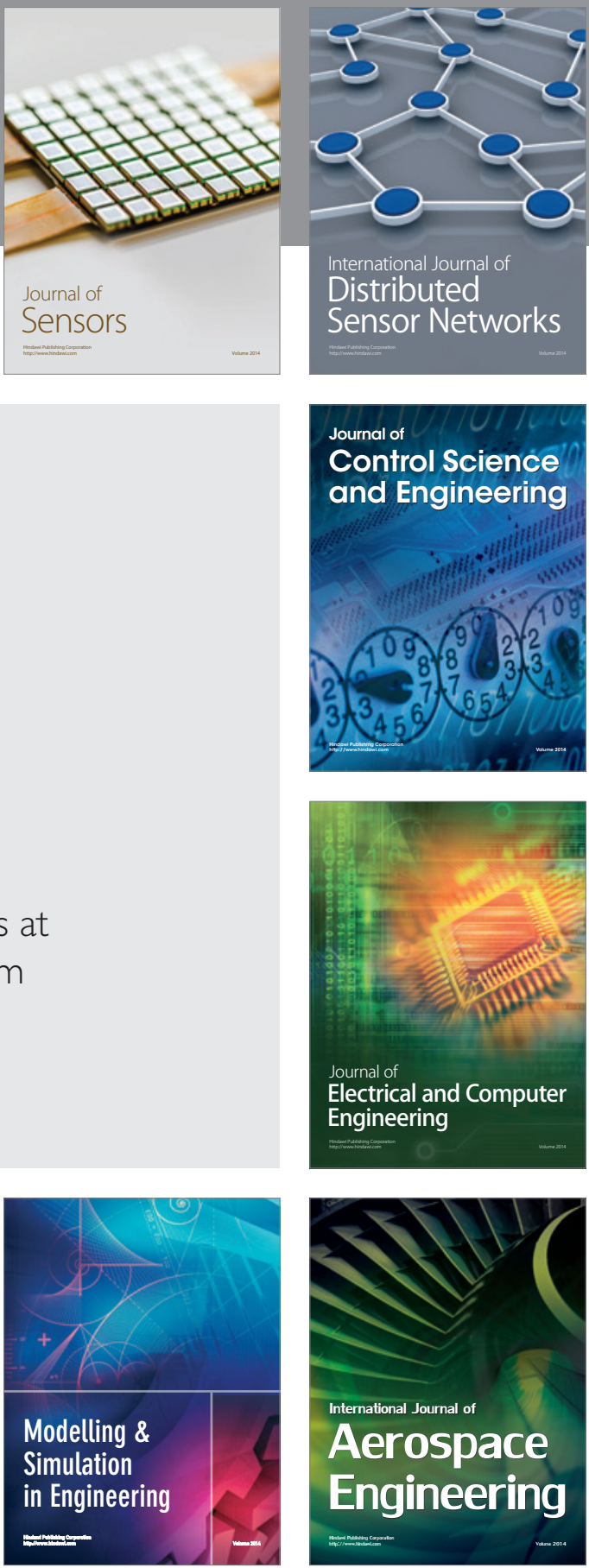

Journal of

Control Science

and Engineering
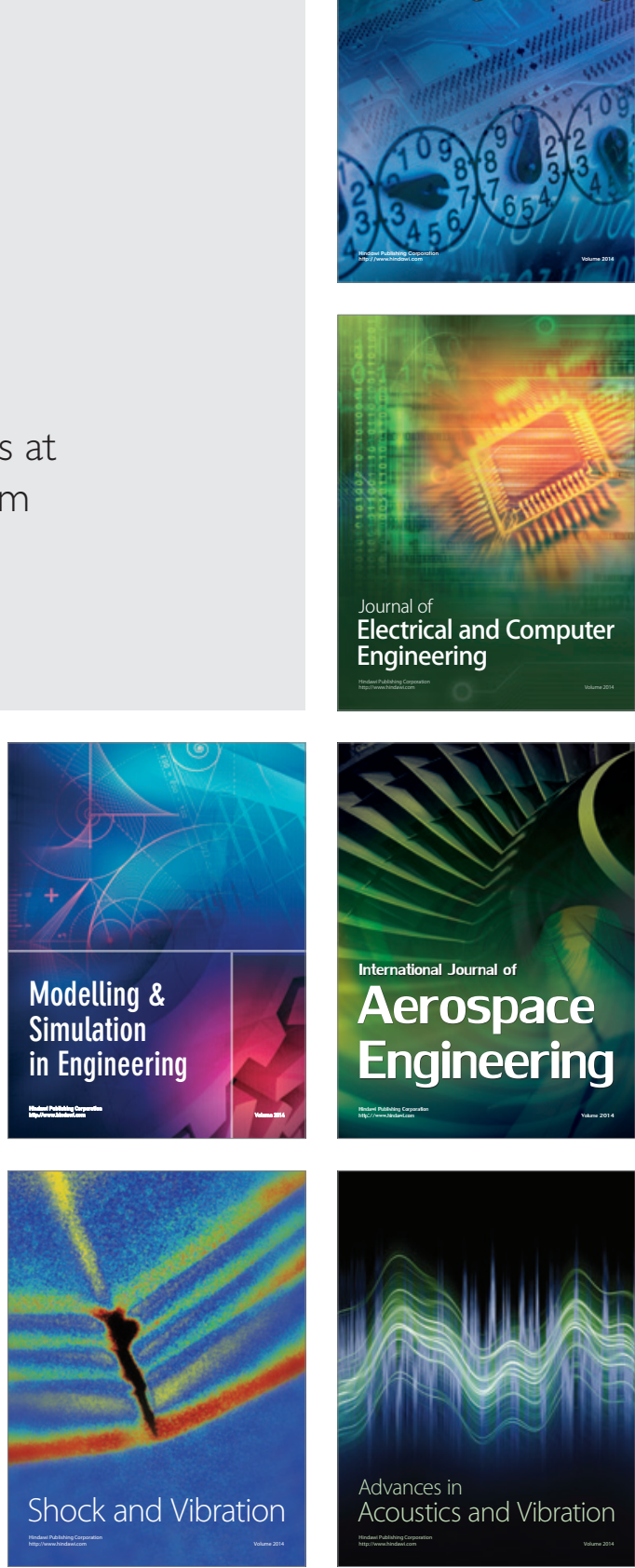